\title{
The prospect of immunotherapy combined with chemotherapy in patients with advanced non-small cell lung cancer: a narrative review
}

\author{
Xiangu Ning ${ }^{1 \#}$, Yang Yu ${ }^{1 \#}$, Songjun Shao ${ }^{2}$, Rong Deng ${ }^{3}$, Jie Yu ${ }^{3}, X^{\prime}$ uming Wang ${ }^{4}, X_{\text {Xeke She }}^{5}$, \\ Depei Huang ${ }^{5}$, Xudong Shen ${ }^{5}$, Weiming Duan ${ }^{5}, J_{i n}$ Duan $^{6}$, Hushan Zhang ${ }^{5} \wedge$
}

${ }^{1}$ Department of Thoracic Surgery, The First People's Hospital of Yunnan Province, Kunming, China; ${ }^{2}$ Department of Respiratory and Critical Medicine, Guizhou Provincial People’s Hospital, Guiyang, China; ${ }^{3}$ Department of Oncology, Guizhou Provincial People’s Hospital, Guiyang, China; ${ }^{4}$ Department of Pulmonary and Critical Care Medicine, The First Affiliated Hospital of Kunming Medical University, Kunming, China; ${ }^{5}$ The Medical Department, 3D Medicines Inc., Shanghai, China; ${ }^{6}$ Department of Geriatric Thoracic Surgery, The First Affiliated Hospital of Kunming Medical University, Kunming, China

Contributions: (I) Conception and design: H Zhang; (II) Administrative support: J Duan, X Ning; (III) Provision of study materials or patients: Y Yu, S Shao, R Deng, J Yu, X Wang; (IV) Collection and assembly of data: H Zhang, X Wang, X She, D Huang, X Shen, W Duan; (V) Data analysis and interpretation: H Zhang; (VI) Manuscript writing: All authors; (VII) Final approval of manuscript: All authors.

\#These authors contributed equally to this work.

Correspondence to: Hushan Zhang. Building 2, Block B, 158 XinJunhuan Street, Pujiang Hi-tech Park, Min Hang District, Shanghai 201114, China. Email: 15111010041@fudan.edu.cn; Jin Duan. No. 295 Xichang Road, Kunming 650031, China. Email: djames310@163.com.

Objective: Based on a thorough analysis of monotherapy (pembrolizumab) and chemoimmunotherapy, the immunomodulatory effects of chemotherapy agents are emphasized.

Background: The combination of chemotherapy and immune checkpoint inhibitors should and is already being regarded as a new standard strategy for the first-line treatment of advanced NSCLC. As some scientists hold, chemoimmunotherapy is the beginning of a new era of lung cancer therapy. Scientists of this field are trying to make the perfect blend, that is, to explore the perfect condition for combination therapy. However, first, we should fully understand the specific role of chemotherapy agents in combination therapy and its specific mechanism. However, our current consideration of this aspect is not comprehensive enough. Based on a full understanding of the mechanisms and roles of these partner treatments, can the perfect blend or a more appropriate combination strategy of cancer immunotherapy be established?

Methods: Search and discuss the literature of pembrolizumab in the treatment of non-small cell lung cancer, as well as previous studies on the immune regulatory function of chemotherapeutic agents, to analyze the mechanism of chemotherapeutic agents in combination immunotherapy.

Conclusion: Here, we carefully analyzed the details of clinical trials of pembrolizumab in the treatment of NSCLC, and reviewed literature in this field. Therefore, we aim to put forward that chemoimmunotherapy is not a simple model of one plus another. Accordingly, we believe that the more likely role of chemotherapeutics in combination therapy with pembrolizumab is an immunomodulator. Based on this perspective, we propose that more attention and efforts should be devoted to understanding and exploring the immunomodulatory function of chemotherapy agents.

Keywords: Chemotherapy; pembrolizumab; immune regulation; adverse events

Submitted Sep 03, 2021. Accepted for publication Nov 04, 2021.

doi: $10.21037 / \mathrm{atm}-21-4878$

View this article at: https://dx.doi.org/10.21037/atm-21-4878

^ ORCID: 0000-0002-7557-4426. 


\section{Introduction}

In recent years, dramatic changes have also been undergone in treating patients with advanced or metastatic nonsmall cell lung cancer (NSCLC). Especially, using immunotherapy has opened a new era in lung cancer treatment. Immunotherapy of immune checkpoint inhitor (ICI) treatment is a approaches to against cancer immune evasion. This is conceptually different from the immune enhancement approach, it aims to restore a lost antitumor immunity, Chen et al. termed it "normalization cancer immunotherapy" (1). Evaluations of the immunerelated response criteriaare vital improtant. The classic RECIST remains a meaningful and reseasonable criteria to assess response to ICI treatment. but immune-related response criteria and systematic criteria should also be put into account, that attempt to capture additional response patterns observed with immune therapy beyond those described by RECIST or WHO criteria. iRECIST, the consensus guideline was developed in cancer immunotherapy trials. Changes in tumour burden, survival and quality of life, adverse event, specifically immunerelated adverse event all were index that used to assess immunotherapy (2-4). Although immunotherapy that targeted the programmed death-1 (PD-1) pathway has gained considerable interest, and achieved remarkable success in the suitable population, for instance, patients with tumor proportions score at least $50 \%$ (TPS $\geq 50 \%$ ), as shown in 5-year results in the updated keynote 024 (5). ICI treatment showed unprecedented durability, Responses to ICI can even last years. while the response rate to ICI single agent in unselected patients in some solid tumors is range from $40-60 \%$, while the response rates in most other tumor types were at a low level, including NSCLC $(6,7)$. in addition, even among patients who initially respond to ICIs, resistance to ICI can eventually develop. In short, only a minority of patients achieve durable response (8). The urgent challenge is to transform most patients from immunotherapy non-responders to responders. This may require potent combination immunotherapies that effectively reshape the immune microenvironment into a more suitable pattern for immunotherapy. In addition, combination therapy and more other new strategies are needed to treat ICI-resistant NSCLC, for examples, ntinostat plus pembrolizumab, that is epigenetic therapies combined with PD-1 blockade to overcome resistance (9). Chemo-immunotherapy combination may be a promising option, as the final results of keynote 189 updated recently (10). From this perspective, it is essential to understand the original characteristics and mechanisms of the tumor microenvironment (TME) in patients who do not respond to immune checkpoint inhibitors (ICIs) and develop effective agents that can modulate tumor immune microenvironment (TIME) to be suitable for ICI therapy. Through retrospective analysis of the results of previous keynote series studies on NSCLC, we concluded and inferred that chemotherapy agents have potential effects during combination therapy, and can maximize the efficacy of combination therapy. Herein, based on data analysis of previous clinical trials of pembrolizumab in the treatment of NSCLC and reviewed literatures in this filed, and hold the perspective that chemotherapy agent role as immunomudulator in combination therapy with pembrolizumab. Therefore, adjusting the appropriate chemotherapy regimen, chemotherapy cycle, dosage, etc. may be a promising direction in the future. We present the following article in accordance with the Narrative Review reporting checklist (available at https://dx.doi.org/10.21037/ atm-21-4878).

\section{Pembrolizumab monotherapy and combination therapy in NSCLC}

Due to the success in the keynote 010 (11), the application of pembrolizumab in the first-line treatment of NSCLC patients has been further explored. Clinical trial keynote 024 (12) showed revolutionary meaning in the treatment of advanced NSCLC patients. The results of this clinical trial reshaped the first-line treatment of patients with advanced NSCLC. Pembrolizumab monotherapy was superior to chemotherapy in patients with tumor proportions score at least $50 \%$ (TPS $\geq 50 \%$ ). The encouraging 5 -year overall survival (OS) updated results of keynote 024 were reported recently that during the 5-year follow-up period, pembrolizumab as a single agent displayed a longer curative effect than platinum-containing chemotherapy. OS rate at 5 -year was $31.9 \%$ vs. $16.3 \%$, the median duration of response was 29.1 vs. 6.3 months in pembrolizumab and chemotherapy, respectively (5).

Based on the encouraging results of the Keynote 024 clinical trial, the Keynote 042 further investigated outcome of pembrolizumab therapy in patients with PD-L1 TPS $>1 \%$ and obtained positive results on the whole population (13). The results of Keynote 042 further supported testing of PD-L1 TPS and using those percentages to guide treatment recommendations. 
Table 1 Adverse events in monotherapy and combination therapy

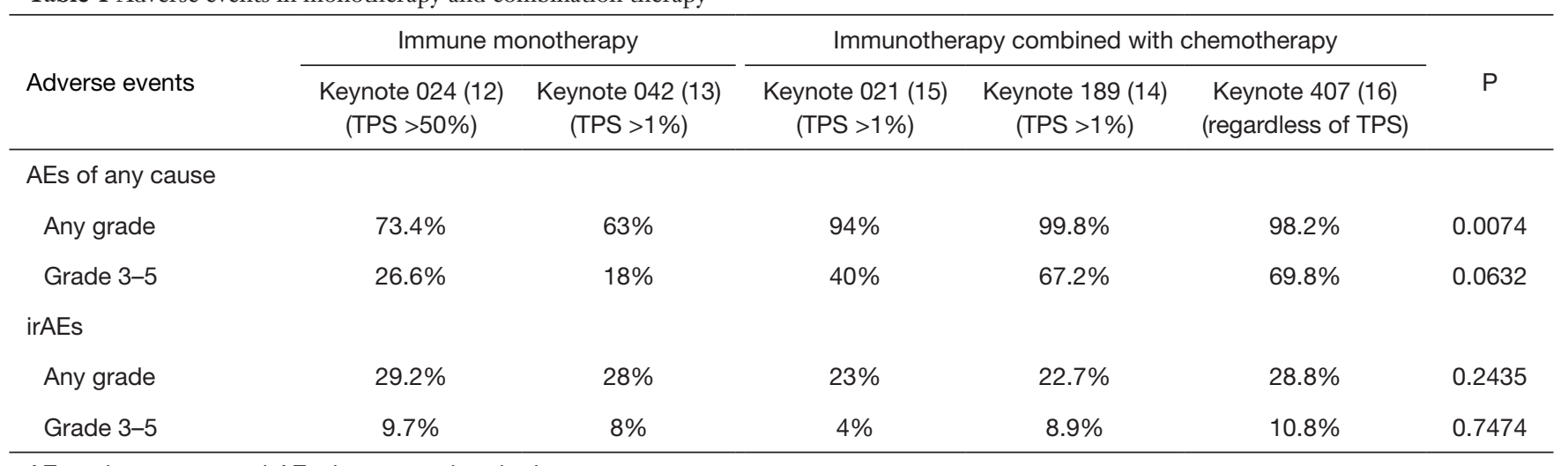

AEs, adverse events; irAEs, immune-related adverse events.

After analyzing the details of the studies, we found that in the first six months of treatment, immunotherapy did not show more obvious superiority than chemotherapy in keynote 024. However, in keynote 042, compared with chemotherapy, significantly poorer results were observed in the first six months of the treatment in the pembrolizumab monotherapy cohort, especially progression-free survival (PFS). These findings suggested that more patients may not benefit from pembrolizumab. Even abnormal progression or hyper-progressive disease (HPD) occurred among these patients in the early stages of clinical trials. Besides, adverse events occurred more in the pembrolizumab monotherapy group $(28 \%$ and $7 \%$ of any grade adverse events in the pembrolizumab and chemotherapy groups, respectively). However, the author did not explain whether this was related to worse performance in OS or PFS in the first six months of the pembrolizumab monotherapy cohort, and it may need further exploration.

Surprisingly, Keynote 189 provided some clues. Pembrolizumab plus chemotherapy prolonged OS and PFS significantly in Keynote 189 trial. Most importantly, compared with the results of the Keynote 042, in the Keynote 189, the combination therapy group benefited more than the chemotherapy group throughout the clinical trial process, and the rates of adverse events were similar in both groups $(14,15)$. This phenomenon is not very clearly displayed in keynote 024 due to different TPS of PD-L1; this means that in keynote 042, more patients are originally not suitable for immunotherapy, while these patients may be more suitable for combination therapy. What is more, comparing the adverse events that occurred in pembrolizumab monotherapy and combination therapy, the results showed no significant differences were observed in immune-related adverse events. Simultaneously, there is a tendency for significant differences in the adverse events of any cause (Table 1). This may imply that combination therapy did not increase adverse events, at least not immune-related adverse events. Furthermore, in phase 2 study keynote 021 (15), patients with previously untreated, advanced NSCLC without EGFR/ALK aberrations were randomized to the pembrolizumab plus chemotherapy group, and the chemotherapy alone group, similar trend as in keynote 189 , was displayed in keynote 021 , especially in PFS, that patients in the combination therapy group gained clinical benefit from the beginning of treatment. These results collectively suggest to a certain extent that chemoimmunotherapy combination strategy can overcome the situation where pembrolizumab monotherapy is not applicable.

\section{Combination immunotherapy and role of chemotherapy}

The antagonists of PD-1/PD-L1 displayed a notable ability to unleash $\mathrm{T}$ cells to present in the TME, termed "immune normalization" by Sanmamed et al. (1). However, infiltration and function of $\mathrm{T}$ cells were affected by complicated mechanisms and factors in TME. For this reason, developing a strategy for profiling the TME to predict clinic response, avoid immune suppression, and escape is the key to improving the clinical efficacy benefit from immunotherapy. Regulatory $\mathrm{T}$ cells (Tregs), myeloidderived suppressor cells (MDSCs), and tumor-associated macrophages (TAM), which accumulated during tumor progression, have been proved to be the main factors associated with immune suppression in the TME. In addition, other inhibitory immune checkpoints, including 
LAG-3, TIM-3, B7-H3, TIGIT, are also factors related to immune suppression in the TME (17). That is, regulation of these factors and mechanisms will facilitate response to antiPD-1/PD-L1 and improve the efficacy of immunotherapy. In other words, it is important to find agents or treatment strategies that can convert an immune cold into an immune hot tumor. Combination immunotherapies that transform non-responders to responders are in rapid development. For example, the clinic trial REGONIVO, in which patients with microsatellite stable (MSS) or mismatch repair-proficient (pMMR) tumors received treatment of regorafenib plus nivolumab, gained inspiring results (18). In this combination therapy, the role and mechanism of regorafenib was anti-angiogenesis and regulation of the immune microenvironment (19). Moreover, chemotherapy also has the potential to convert an immune cold tumor into a hot one, and may thus lead to synergy with anti-PD-1/ PD-L1.

On the one hand, chemotherapy agents can promote tumor antigen presentation and prime neoantigen-specific T-cell responses; alternatively, they can even inhibit immune suppressive factors (20). Chemoimmunotherapy combinations are performing and potentially changing the current strategy of metastatic NSCLC treatment for their superior performances, as shown in the clinical trial results keynote 021 and keynote 189 mentioned above. Through synergy, chemotherapy agents and pembrolizumab combination therapy displayed superior performances in PFS and OS, compared with chemotherapy. When comparing the results of keynote 189 and keynote 042 , we can find that combined immunotherapy has been shown to overcome more adverse events and poorer responses during the first six months of treatment. The only obvious difference of baseline characteristics is that the non-squamous NSCLC in keynote 042 and 189 was $61.4 \%$ and $100 \%$, respectively. The keynote 407 is another chemoimmunotherapy combination clinical trial in which squamous NSCLC patients were recruited (16). When compared with keynote 042 , the results of keynote 407 also showed a similar trend with the keynote 021 and keynote 189 ; in detail, the combination group showed clinical benefit at the beginning of treatment. Therefore, we speculate that chemotherapy agents may show direct cytotoxicity and play a synergistic effect with anti-PD-1/PD-L1 by regulating the immune microenvironment, which is paving the way for anti-PD-1/PD-L1 therapy.

Researchers found that chemotherapy displayed Immunomodulatory function, for example, induction of
$\mathrm{CD} 8^{+} \mathrm{T}$ cell recruitment to the tumor microenvironment $(21,22)$ and regulation of tumor-associated macrophage (23). A recent study showed that leukemia stem cells (LSCs) promote tumorigenesis and resistance to therapy under chemotherapy-induced stress. Doxorubicin (DXR) plays a role as an inhibitor of the Akt- $\beta$-catenin interaction at low doses and inhibits expression of multiple immune checkpoints, including PD-L1, CD24, and TIM3. Therefore, overcoming cancer therapy resistance and immune escape (24). If considering combination therapy or pembrolizumab monotherapy as the main difference between the keynote 042 and 189 , the immunomodulatory effect of chemotherapy can explain the phenomenon mentioned above to a certain extent. That is, chemotherapy agents reshaped the immune microenvironment of patients, even turned the cold immune tumor into a hot tumor. Therefore, chemotherapy paved the way for pembrolizumab.

Besides, Some studies demonstrated metastatic patterns seen among several of cancers, and it showed that metastasis of NSCLC patients is the main cause of recurrence and death $(25,26)$. Lymphatic metastasis is the main route of NSCLC metastasis, therefore, regulation of lymphatic activity, so called immune factors such as cytokines and chemokines would affect metastasis (27). As reported, CCR7-CCL2 1 axis mediate metastasis and progression of NSCLC (26). So far, little is known about whether chemotherapeutics have the ability to regulate chemokines, and some attention should be paid in this direction to regulate the metastasis of NSCLC.

\section{Safety of therapeutic monoclonal antibody including ICI}

Monoclonal antibodies (mAbs) are developed as therapeutic strategy for autoimmune and infectious diseases, transplant rejection, and malignancies, even a range of new indications. However, application of mAbs is a double-edge sward as it carries the risk of a series of immune reactions, including serum sickness, acute anaphylaxis etc. what's more, the administration of mAbs accompanied by numerous adverse effects that are related to their specific targets such as autoimmune disease, cancer or organ-specific adverse events $(28,29)$. In addition to targeting cancer antigens antibodies can also modulate immunological pathways, for example, most therapeutic mAbs can effectively mediates complement fixation and antibody-dependent cellular cytotoxicity (ADCC) (29). 
After introduction and approval of anti-vascular endothelial growth factor antibody such as bevacizumab, and antiepidermal growth factor antibody such as cetuximab, the most significant advances in recent therapy of solid tumors should be the application of antibody therapy directed against several immune checkpoints. ICI has led to durable response in some patients, but may also accompanied with immune-related adverse events (irAEs), which are similar in nature to autoimmune diseases. IrAEs include immuneconditioned colitis and diarrhea,dermal toxicity,hepatic toxicity,endocrinopathy, and other inflammatory events such as meningitis, pneumonitis, uveitis, pancreatitis, myocarditis, pericarditis, nephritis. Because irAEs may occured due to general immunologic enhancement, temporary immunosuppression with tumor necrosis factor $\alpha$ antagonists, corticosteroids or other agents is often recommended to control irAE (30-32).

\section{PD-L1 expression and ICI efficacy}

Multiple clinical trials have demonstrated positive correlation between clinical efficacy of monotherapg and level of PD-L1 expression. In particular, KEYNOYE-024, demonstrated superior OS for pembrolizumab in patients with PD-L1 $\geq 50 \%(12,33)$. In second-line clinical trials, although greatest benefits were observed in tumors with higher PD-L1 expression, it was demonstrated in all subjects that patients were treated with either atezolizumab or nivolumab had longer survival than chemotherapy (34). While results of keynote 021 and keynote 189 studies suggested that NSCLC patients benefited from pembrolizumab combined with chemotherapy, irrespective of PD-L1 expression $(14,15)$. For difference in the efficacy of different ICI, a meta analysis revealed that PD-1 mAbs exhibited superior PFS (HR, 0.73; 95\% CI: 0.56-0.96; $\mathrm{P}=0.02)$ and $\mathrm{OS}(\mathrm{HR}, 0.75$; 95\% CI: $0.65-0.86$; $\mathrm{P}<0.001$ ) compared with PD-L1 mAbs, while no significant difference wasfound in safety profile (35). Another meta analysis suggested that anti-CTLA-4 and anti-PD-1 have different irAE profiles. Grade 3/4 irAE were more common anti-CTLA-4 compared with anti-PD-1 therapy. All grades hypophysitis, colitis and rash were more frequent with antiCTLA-4; while hypothyroidism, pneumonitis and arthralgia were more common with anti-PD-1 therapy (36).

\section{Conclusions}

Both chemotherapy and immunotherapy are important treatment options for advanced NSCLC patients. However, the response rate of monotherapy is still low. Combination immunotherapy may be potential strategy to expand the benefit population and improve the survival benefit in NSCLC. And some researched have been explored recent years, such as immunotherapy combined with chemotherapy (14), radiotherapy (37), antiangiogenesis (38), targeted therapy (39), and anti-PD-1/PD-L1 plus anti-CTLA-4 etc $(40,41)$. Data from clinical trials of combination therapy suggested that these combination strategies showed obvious efficacy and without significant increase in toxicity. What's more, combination therapy of anti-PD-1 and cheotherapy has been approved by FDA in first line treatment for metastatic NSCLC without driver mutation $(42,43)$. Chemo-immunotherapy combination therapy is currently being evaluated as a promising treatment, but it is not simply the one plus one. It is more important to clarify the mechanism behind the combination therapy and determine the synergy mode between the chemotherapy agents and pembrolizumab. To warrant a future combination of immunotherapy with the standardof-care therapy to improve clinical benefit in patients with cancer, the role and mechanism of chemotherapy agents in the combination therapy that beyond directing anti-tumor activity needs further exploration. Based on the detailed analysis and summary of the above studies, we infer that chemotherapy agents may play a role as the immune modulator or normalizer in combination therapy to clear the way and create suitable conditions for immunotherapy agents, such as pembrolizumab. We need to re-evaluate the chemotherapy regimen and adjust the most appropriate chemotherapy drug formulation, drug dosage, and medication cycle to play a greater role in combination therapy. Furthermore, current challenges facing immunotherapy are finding optimal combination strategy and agents other than chemo-immune combination to achieve better therapeutic synergy and improve clinical benefit.

\section{Acknowledgments}

Funding: None.

\section{Footnote}

Reporting Checklist: The authors have completed the Narrative Review reporting checklist. Available at https:// dx.doi.org/10.21037/atm-21-4878 
Conflicts of Interest: All authors have completed the ICMJE uniform disclosure form (available at https://dx.doi. org/10.21037/atm-21-4878). HZ, DH, XS, XS and WD are employed by 3D Medicines Inc. The other authors have no conflicts of interest to declare.

Ethical Statement: The authors are accountable for all aspects of the work in ensuring that questions related to the accuracy or integrity of any part of the work are appropriately investigated and resolved.

Open Access Statement: This is an Open Access article distributed in accordance with the Creative Commons Attribution-NonCommercial-NoDerivs 4.0 International License (CC BY-NC-ND 4.0), which permits the noncommercial replication and distribution of the article with the strict proviso that no changes or edits are made and the original work is properly cited (including links to both the formal publication through the relevant DOI and the license). See: https://creativecommons.org/licenses/by-ncnd $/ 4.0 \%$.

\section{References}

1. Sanmamed MF, Chen L. A Paradigm Shift in Cancer Immunotherapy: From Enhancement to Normalization. Cell 2018;175:313-26.

2. Borcoman E, Kanjanapan Y, Champiat S, et al. Novel patterns of response under immunotherapy. Ann Oncol 2019;30:385-96.

3. Seymour L, Bogaerts J, Perrone A, et al. iRECIST: guidelines for response criteria for use in trials testing immunotherapeutics. Lancet Oncol 2017;18:e143-52.

4. Wolchok JD, Hoos A, O'Day S, et al. Guidelines for the evaluation of immune therapy activity in solid tumors: immune-related response criteria. Clin Cancer Res 2009;15:7412-20.

5. Reck M, Rodríguez-Abreu D, Robinson AG, et al. Five-Year Outcomes With Pembrolizumab Versus Chemotherapy for Metastatic Non-Small-Cell Lung Cancer With PD-L1 Tumor Proportion Score $\geq 50$. J Clin Oncol 2021;39:2339-49.

6. Antonia SJ, Borghaei H, Ramalingam SS, et al. Four-year survival with nivolumab in patients with previously treated advanced non-small-cell lung cancer: a pooled analysis. Lancet Oncol 2019;20:1395-408.

7. Fradet Y, Bellmunt J, Vaughn DJ, et al. Randomized phase III KEYNOTE-045 trial of pembrolizumab versus paclitaxel, docetaxel, or vinflunine in recurrent advanced urothelial cancer: results of $>2$ years of follow-up. Ann Oncol 2019;30:970-6.

8. Schoenfeld AJ, Hellmann MD. Acquired Resistance to Immune Checkpoint Inhibitors. Cancer Cell 2020;37:443-55.

9. Hellmann MD, Jänne PA, Opyrchal M, et al. Entinostat plus Pembrolizumab in Patients with Metastatic NSCLC Previously Treated with Anti-PD-(L)1 Therapy. Clin Cancer Res 2021;27:1019-28.

10. Rodriguez-Abreu D, Powell SF, Hochmair MJ, et al. Pemetrexed plus platinum with or without pembrolizumab in patients with previously untreated metastatic nonsquamous NSCLC: protocol-specified final analysis from KEYNOTE-189. Ann Oncol 2021;32:881-95.

11. Herbst RS, Baas P, Kim DW, et al. Pembrolizumab versus docetaxel for previously treated, PD-L1-positive, advanced non-small-cell lung cancer (KEYNOTE-010): a randomised controlled trial. Lancet 2016;387:1540-50.

12. Reck M, Rodríguez-Abreu D, Robinson AG, et al. Pembrolizumab versus Chemotherapy for PD-L1Positive Non-Small-Cell Lung Cancer. N Engl J Med 2016;375:1823-33.

13. Mok TSK, Wu YL, Kudaba I, et al. Pembrolizumab versus chemotherapy for previously untreated, PD-L1expressing, locally advanced or metastatic non-small-cell lung cancer (KEYNOTE-042): a randomised, open-label, controlled, phase 3 trial. Lancet 2019;393:1819-30.

14. Gandhi L, Rodríguez-Abreu D, Gadgeel S, et al. Pembrolizumab plus Chemotherapy in Metastatic NonSmall-Cell Lung Cancer. N Engl J Med 2018;378:2078-92.

15. Langer CJ, Gadgeel SM, Borghaei H, et al. Carboplatin and pemetrexed with or without pembrolizumab for advanced, non-squamous non-small-cell lung cancer: a randomised, phase 2 cohort of the open-label KEYNOTE-021 study. Lancet Oncol 2016;17:1497-508.

16. Paz-Ares L, Luft A, Vicente D, et al. Pembrolizumab plus Chemotherapy for Squamous Non-Small-Cell Lung Cancer. N Engl J Med 2018;379:2040-51.

17. Shimizu K, Iyoda T, Okada $M$, et al. Immune suppression and reversal of the suppressive tumor microenvironment. Int Immunol 2018;30:445-54.

18. Fukuoka S, Hara H, Takahashi N, et al. Regorafenib Plus Nivolumab in Patients With Advanced Gastric or Colorectal Cancer: An Open-Label, Dose-Escalation, and Dose-Expansion Phase Ib Trial (REGONIVO, EPOC1603). J Clin Oncol 2020;38:2053-61.

19. Arai H, Battaglin F, Wang J, et al. Molecular insight of regorafenib treatment for colorectal cancer. Cancer Treat 
Rev 2019;81:101912.

20. Zitvogel L, Kepp O, Kroemer G. Immune parameters affecting the efficacy of chemotherapeutic regimens. Nat Rev Clin Oncol 2011;8:151-60.

21. Gao Q, Wang S, Chen X, et al. Cancer-cell-secreted CXCL11 promoted CD8+ T cells infiltration through docetaxel-induced-release of HMGB1 in NSCLC. J Immunother Cancer 2019;7:42.

22. Heeren AM, van Luijk IF, Lakeman J, et al. Neoadjuvant cisplatin and paclitaxel modulate tumor-infiltrating $\mathrm{T}$ cells in patients with cervical cancer. Cancer Immunol Immunother 2019;68:1759-67.

23. Wanderley CW, Colón DF, Luiz JPM, et al. Paclitaxel Reduces Tumor Growth by Reprogramming TumorAssociated Macrophages to an M1 Profile in a TLR4Dependent Manner. Cancer Res 2018;78:5891-900.

24. Perry JM, Tao F, Roy A, et al. Overcoming Wnt- $\beta$-catenin dependent anticancer therapy resistance in leukaemia stem cells. Nat Cell Biol 2020;22:689-700.

25. Disibio G, French SW. Metastatic patterns of cancers: results from a large autopsy study. Arch Pathol Lab Med 2008;132:931-9.

26. Zhang S, Wang H, Xu Z, et al. Lymphatic Metastasis of NSCLC Involves Chemotaxis Effects of Lymphatic Endothelial Cells through the CCR7-CCL21 Axis Modulated by TNF- $\alpha$. Genes (Basel) 2020;11:1309.

27. Vilgelm AE, Richmond A. Chemokines Modulate Immune Surveillance in Tumorigenesis, Metastasis, and Response to Immunotherapy. Front Immunol 2019;10:333.

28. Buss NA, Henderson SJ, McFarlane M, et al. Monoclonal antibody therapeutics: history and future. Curr Opin Pharmacol 2012;12:615-22.

29. Hansel TT, Kropshofer H, Singer T, et al. The safety and side effects of monoclonal antibodies. Nat Rev Drug Discov 2010;9:325-38.

30. Day D, Hansen AR. Immune-Related Adverse Events Associated with Immune Checkpoint Inhibitors. BioDrugs 2016;30:571-84.

31. Demlova R, Valík D, Obermannova R, et al. The safety of therapeutic monoclonal antibodies: implications for cancer therapy including immuno-checkpoint inhibitors. Physiol Res 2016;65:S455-62.

32. Weber JS, Kähler KC, Hauschild A. Management of immune-related adverse events and kinetics of response with ipilimumab. J Clin Oncol 2012;30:2691-7.

33. Reck M, Rodríguez-Abreu D, Robinson AG, et al. Updated Analysis of KEYNOTE-024: Pembrolizumab
Versus Platinum-Based Chemotherapy for Advanced NonSmall-Cell Lung Cancer With PD-L1 Tumor Proportion Score of 50\% or Greater. J Clin Oncol 2019;37:537-46.

34. Bodor JN, Boumber Y, Borghaei H. Biomarkers for immune checkpoint inhibition in non-small cell lung cancer (NSCLC). Cancer 2020;126:260-70.

35. Duan J, Cui L, Zhao X, et al. Use of Immunotherapy With Programmed Cell Death 1 vs Programmed Cell Death Ligand 1 Inhibitors in Patients With Cancer: A Systematic Review and Meta-analysis. JAMA Oncol 2020;6:375-84.

36. Khoja L, Day D, Wei-Wu Chen T, et al. Tumour- and class-specific patterns of immune-related adverse events of immune checkpoint inhibitors: a systematic review. Ann Oncol 2017;28:2377-85.

37. Shaverdian N, Lisberg AE, Bornazyan K, et al. Previous radiotherapy and the clinical activity and toxicity of pembrolizumab in the treatment of non-small-cell lung cancer: a secondary analysis of the KEYNOTE-001 phase 1 trial. Lancet Oncol 2017;18:895-903.

38. Manegold C, Dingemans AC, Gray JE, et al. The Potential of Combined Immunotherapy and Antiangiogenesis for the Synergistic Treatment of Advanced NSCLC. J Thorac Oncol 2017;12:194-207.

39. Ahn MJ, Sun JM, Lee SH, et al. EGFR TKI combination with immunotherapy in non-small cell lung cancer. Expert Opin Drug Saf 2017;16:465-9.

40. Hellmann MD, Paz-Ares L, Bernabe Caro R, et al. Nivolumab plus Ipilimumab in Advanced Non-Small-Cell Lung Cancer. N Engl J Med 2019;381:2020-31.

41. Reck M, Borghaei H, O'Byrne KJ. Nivolumab plus ipilimumab in non-small-cell lung cancer. Future Oncol 2019;15:2287-302.

42. Li JX, Huang JM, Jiang ZB, et al. Current Clinical Progress of PD-1/PD-L1 Immunotherapy and Potential Combination Treatment in Non-Small Cell Lung Cancer. Integr Cancer Ther 2019;18:1534735419890020.

43. Marmarelis ME, Aggarwal C. Combination Immunotherapy in Non-small Cell Lung Cancer. Curr Oncol Rep 2018;20:55.

Cite this article as: Ning $\mathrm{X}$, Yu Y, Shao S, Deng R, Yu J, Wang X, She X, Huang D, Shen X, Duan W, Duan J, Zhang H. The prospect of immunotherapy combined with chemotherapy in patients with advanced non-small cell lung cancer: a narrative review. Ann Transl Med 2021;9(22):1703. doi: 10.21037/atm$21-4878$ 